\title{
Synthesis of Uniform Silica Rods, Curved Silica Wires and Silica Bundles Using Filamentous Fd Virus as a Template
}

\author{
Zhenkun Zhang and Johan Buitenhuis*
}

[*] Z. Zhang, Dr. J. Buitenhuis

Forschungszentrum Jülich GmbH, IFF-Weiche Materie, D-52425 Jülich (Germany)

Fax: +492461612280

E-mail: j.buitenhuis@,fz-juelich.de

[**] The authors thank Jochen Friedrich for his assistance with the TEM imaging and EDAX experiments. Karin Sellinghoff is thanked for her assistance with the fd virus preparation and Pavlik Lettinga for his encouraging discussions.

One dimensional anisotropic inorganic nanostructures such as tubes, rods, wires, fibers, etc. are in the focus of research interests due to their potential applications, for example in optical, electronic and mechanical devices, sensors and catalysis ${ }^{[1,2]}$. The synthesis of these anisotropic nanostructures is a big challenge, because most inorganic materials do not form the desired structure by themselves. In contrast to inorganic systems, biological and organic materials, especially supramolecular systems, usually have a well defined structure down to the nano-scale. Using (bio)organic materials as a template to build up anisotropic inorganic nanostructures has therefore emerged as a highly attractive method in recent years ${ }^{[3]}$. The results described in the present paper may serve as a basis for the further development of the synthesis of inorganic materials using biopolymers as a template. Similar routes of synthesis occur spontaneously in nature, for instance, in the formation of the silica cell wall of the Diatom, which is formed with an amazingly perfect pattern $^{[4]}$. For the synthesis of nanomaterials, rod-like viruses ${ }^{[5-8]}$, carbon nanotubes $^{[9,10]}$, 
lipid nanotubes ${ }^{[11,12]}$, rod-like structures formed from organogelators ${ }^{[13,14]}$, single $\mathrm{DNA}^{[15]}$ etc. offer many templates to direct the formation of anisotropic inorganic materials with special structures and properties.

In this paper, the filamentous fd virus is used as a template to regulate the formation of silica nanomaterials with well-defined morphologies. Fd viruses have a length of $880 \mathrm{~nm}$ and a diameter of $6.6 \mathrm{~nm}^{[16]}$. Each virus consists of a single-stranded DNA molecule packed in a sheath of 2,700 identical coating proteins ${ }^{[17]}$. Besides of its role in biochemistry where it is used for molecular cloning ${ }^{[18]}$, fd has also been used as a rod-like model system to understand some fundamental problems of complex fluids, because it is monodisperse in size and has a nearly hard rod conformation (persistence length $2.2 \mu \mathrm{m})^{[19]}$. M13, a virus which is almost identical to fd, differing only in one amino acid per coating protein, has been intensively explored by Belcher, Hammond and co-worker as a template in the synthesis of metallic and other magnetic and semiconducting nanowires $^{[20-23]}$. Their strategy is to modify the coat protein of M13 via genetic engineering specifically for each metal or oxide, so that the coat protein can selectively induce precipitation or assembly of that specific metal or oxide on the surface of the virus. However, as far as we know, there is no report concerning the application of fd or M13 as a template for silica precipitation.

Another rod-like virus that has been used as a template in the preparation of inorganic nanomaterials ${ }^{[5,8,24-26]}$ is the Tobacco Mosaic Virus (TMV). Compared to TMV, fd and M13 are much thinner and longer and therefore they are good candidates for the synthesis of 1D anisotropic inorganic nanostructures. In addition, fd and M13 do not end-to-end 
self-assemble as is the case for TMV. Sometimes this self-assembly will introduce polydispersity into the final materials ${ }^{[5]}$.

In contrast to the complicated genetic engineering route used with the M13 virus, we show here that wild-type fd virus can also be used as a template in the synthesis of inorganic materials using simple sol-gel chemistry. Under different conditions, using acid-catalyzed hydrolyzation and condensation of tetraethoxysilane as silica precursor, three kinds of morphologies are observed: 1) silica nanorods with a diameter of $20 \mathrm{~nm}$ and a homogeneous silica layer, 2) nanowires with a curved shape and 3) bow-tie-shaped bundles with well-defined shape and hierarchy. Furthermore, a water-soluble organic silicate, tetrakis(2-hydroxyethyl) orthosilicate(THEOS) ${ }^{[27]}$, was used to prepare big silica rods. As far as we know, we are the first to use fd as a template for material synthesis and have observed several interesting structures.

Table 1 gives an overview of the various synthesis conditions investigated together with the resulting morphologies. Single silica rods with high uniformity, aggregated silica-virus hybrid nanorods as well as pronounced granular silica are observed for sample $\mathbf{1}$ (Table 1, Figure 1). Along the axis of the rods, the diameter is constant and the silica layer is homogeneous. The surface of these rods is smooth under the maximum resolution of the TEM we used and the shape of the ends of the rods is semi-spherical. Although fd is semi-flexible and somewhat curved in dispersion, most of the rods are straight and only a few slightly curved rods are seen (Figure 1c). From a single rod point of view, the silica coating is highly uniform. Also the diameters of different rods are all close to the average value of about $20 \mathrm{~nm}$. However, large differences in length are seen for different rods. Long rods with a length comparable to the length of intact fd are observed along with short rods, which might form from the silica coating of the fragments of decomposed fd. 
Table 1. Experimental conditions and the resulting morphology of silica coating of fd

\begin{tabular}{|c|c|c|c|c|c|}
\hline No. & Precursor & $\begin{array}{c}\mathrm{C}^{[\mathrm{a}]} \\
\left(\mathrm{mg} \mathrm{mL}^{-1}\right)\end{array}$ & $\begin{array}{c}\mathrm{P} / \mathrm{T}^{[\mathrm{b}]} \\
\left(\mu \mathrm{L} \mathrm{mg}^{-1}\right)\end{array}$ & $\mathrm{pH}^{[\mathrm{c}]}$ & Morphology ${ }^{[d]}$ \\
\hline 1 & TEOS & 6 & $75 / 6$ & 2.32 & Straight $\operatorname{rods}(\sim 20 \mathrm{~nm})$ \\
\hline $1 \mathbf{a}$ & & & & & Bow-tie-shaped bundles \\
\hline 2 & TEOS & 3 & $35.5 / 3$ & 1.90 & $\begin{array}{l}\text { Nanowires, long, entangled, } \\
\text { curved } 23 \mathrm{~nm} \text {-rods with an } \\
\text { irregular, rough surface }\end{array}$ \\
\hline 3 & THEOS & 6 & $75 / 6$ & 2.32 & Long, curved and big rods \\
\hline 4 & TEOS/ethanol ${ }^{[\mathrm{e}]}$ & 6 & $75 / 6$ & 2.75 & Rods, surface is rough \\
\hline
\end{tabular}

[a] Concentration of fd, volume $1 \mathrm{~mL}$; [b] The volume of the precursor $(\mathrm{P}) /$ the mass of the template (T); [c] The values were measured just before the silica precursors were added; [d] Only well defined morphologies are concerned although always a lot of granular silica was found; [e] The amount of ethanol used is $10 \%(\mathrm{v} / \mathrm{v})$ of the total dispersion volume before adding the silica precursor.

Some of the rods show a clear core-shell structure with a low contrast part along the center of the whole rod (Figure 1b). The low contrast part might be fd. However, some rods do not show such low contrast part and look like pure silica rods (Figure 1c). The width of the low contrast part was found to vary between different rods ranging from $1 \sim 5$ $\mathrm{nm}$, always less than that of intact fd, $6.6 \mathrm{~nm}$. The origin of these smaller core diameters is not clear. Maybe some artifact of the imaging process plays a role, or some calcination process caused by the heating of the electron beam in the microscope results in smaller core diameters. The "pure" silica rod shown in Figure 1c was subjected to EDAX analysis. Apart from silicon and oxygen, nitrogen and phosphorus are detected (Figure 
1d). The nitrogen and phosphorus can only be attributed to fd, given that no agents containing nitrogen or phosphorus were used during the synthesis. This could also indicate that the method is destructive to the viruses and therefore the diameters of the cores vary. For instance, some of the rods might only be templated by the (partially) stripped DNA. Nevertheless, the presented results show convincingly that the semiflexible fd virus can act as a template for straight silica rods.

Bow-tie-shaped bundles of silica rods are formed (Figure 2) if the straight rod dispersion 1 (Table 1) is mixed with a methanol/ammonia mixture as described in the experimental section (sample 1a in Table 1). This morphology is remarkable because as far as we know, no similar morphology has been reported for any other virus or organic template in the past. The bundles all have similar dimensions. The maximum length along the y axis of the well-defined bundles is about $2000 \mathrm{~nm}$, comparable to the total length of two intact fd viruses joined with each other head-to-tail (see the cartoon in Figure 2). The main components of all bundles are rods, as illustrated by a defect bundle from which an independent rod with a contour length consistent with that of intact fd protrudes (Figure 3a). Apart from the dominant bow-tie-shaped bundles as shown in Figure 2, sometimes also "single fans" (Figure 2, see arrow), defect bundles (Figure 3a) and cross-like bundles (Figure 3b) are observed. As the bow-tie-shaped bundles are the dominant bundles, we suggest that the cross-like bundles are formed by an aggregation between two bow-tieshaped bundles. The formation of the bow-tie-shaped bundles itself seems to originate from an aggregation of (silica coated) fd viruses (and granular silica) after addition of the methanol/ammonia mixture, but the exact reason for the shape and size of the bundle remains unclear. 
At lower fd concentration and a slightly lower $\mathrm{pH}$ (see sample 2 in Table 1), long, curved wires are observed entangled with each other (Figure 4). The surface of these wires is less smooth than that of the straight rods described before, and the diameter of these wires shows a less sharp distribution with an average diameter of about $23 \mathrm{~nm}$. The contour length of these wires is in the range of that of intact fd, while long wires with a length twice that of fd are also observed. The longer wires seem to consist of two viruses sticking together by partial parallel overlap. These results imply that most fd remains intact during wire formation (an exception is the broken wire shown in the inset of Figure 4 by the white arrow), in contrast to the case of the straight rods described above, where many rods much shorter than fd are observed.

The curved shape of these wires probably originates from the bending configurations of the semi-flexible fd virus in aqueous media. Therefore, these hybrid silica wires show an example of a quite precise transcription of the template, here, semi-flexible fd. Whether or not the silica coating solidifies the fd virus completely so that the flexibility is lost is not clear.

Tetrakis(2-hydroxyethyl) orthosilicate(THEOS) is a highly water-soluble silica precursor $^{[27]}$. When THEOS was used as the source of silica (3 in Table 1) instead of TEOS, big rods with a diameter of about $150 \mathrm{~nm}$ and an irregular surface (Figure 5a) are observed. The length of most of the big rods is between $800 \mathrm{~nm}$ (inset of Figure 5a) and $2000 \mathrm{~nm}$.

When the solubility of the TEOS was increased by adding TEOS together with $10 \%$ of the sample volume of ethanol (4 in Table 1), only straight nanorods are observed (Figure $5 b)$, but in contrast to the rods obtained without ethanol and otherwise identical 
conditions (1 in Table 1, Figure 1), the surface of most of these rods is rough (Figure 5b). Closer examination indicates the presence of small nanoparticles $(2 \mathrm{~nm})$.

A straightforward mechanism is generally accepted to describe the formation of inorganic nanomaterials from alkoxides using organic materials as the template ${ }^{[28,29]}$ : a precursor of the inorganic material hydrolyzes under a catalyst. The hydrolyzed precursors (or monomers) may condense to form dimers, trimers and other oligomers or even small particles with a size of several $\mathrm{nm}$. These intermediate species deposit onto the surface of the template through interactions such as electrostatic attraction, hydrogen bonding, etc. The deposited intermediate species further condense with each other to form the inorganic materials with the morphology of the template.

Studies on the precipitation of silica in the diatom ${ }^{[4]}$ show that cationic amines play an important role. Because each coating protein of the fd virus has one terminal amine and one amine from a lysine residue, which are protonated at the $\mathrm{pH}$ used in our experiments $(\mathrm{pH} \approx 2)$, it might be that these cationic amines show some interaction with the silica. As silica is essentially uncharged at $\mathrm{pH} 2$, charge interactions are unlikely. Maybe, hydrogen bonding between the cationic amines and the silanol groups of the silica is important. However, the essential interaction between silica and fd might also be a more subtle combination of interactions, as is also the case for the diatom. However, for this mechanism to work, the affinity of the surface of the fd virus towards silica deposition has to be high enough, so that heterogeneous nucleation of silica onto the surface of the fd virus can compete with the homogeneous nucleation of silica in the solution (so-called secondary nucleation). In the present study the formation of silica is not limited 
exclusively to the surface of the fd virus, but also a lot of granular silica (Figure 3) due to secondary nucleation is observed.

Furthermore, the amount of silica precursor was taken such that it can form a $1 \mathrm{~nm}$ layer of silica around all fd viruses. Because the actual layer thickness observed is much larger than $1 \mathrm{~nm}$ and in addition part of the silica is lost in the secondary nucleation, this means that many fd viruses are not coated at all. It is not clear why some of the fd viruses are coated with a smooth silica layer with a narrow distribution of the diameter, while some other fd viruses are not coated at all.

We demonstrated the capability of fd viruses to be used as a template for the formation of 1D silica nanomaterials. Three nanostructures with distinct morphologies have been observed under different sol-gel conditions using TEOS as silica precursor: silica rods, wires and bow-tie-shaped bundles. Silica wires seem to transcript the bending conformation and length of intact semi-flexible fd, but under somewhat different reaction conditions also remarkably straight silica rods are formed that have a high uniformity in terms of the thickness and homogeneity of the silica layer. The bow-tie-shaped bundles may form from an aggregation of (silica coated) fd viruses under the conditions adopted here, leading to bundles with a defined dimension and shape. Compared with many other organic templates, fd is almost perfect because of its narrow size distribution and the homogeneous properties of its surface. Work devoted to further understanding the results obtained in this paper and exploring the above problems is ongoing. 


\section{Experimental Section}

All agents were purchased from Sigma-Aldrich except where noted otherwise. Tetraethoxysilane (TEOS) (99\%, Fluka) was freshly distilled. Ammonium hydroxide (25\%, Merck, hereafter referred to as ammonia), methanol (99.9\%) and tetrakis(2hydroxyethyl) orthosilicate(THEOS) solution in ethylene glycol (density: $1.196 \mathrm{~g} \mathrm{~mL}^{-1}$ at $\left.25^{\circ} \mathrm{C}\right)$ were used as received. $\mathrm{HCl}(25 \%)$ and absolute ethanol (99.9\%) were obtained from KMF Laborchemie Handels GmbH. Water from a Millipore Milli-Q10 system was always used.

The fd virus was grown and purified following standard biochemical protocols ${ }^{[18]}$ using the XL 1 blue strain of E. coli as the host bacteria. The standard yield was ca. $15 \mathrm{mg}$ of fd per litre of infected bacteria suspension, and the virus was typically grown in $6 \mathrm{~L}$ batches. After purification, the virus was redispersed in a $10 \mathrm{mM} \mathrm{NaHCO}_{3}$ buffer with $15 \%(\mathrm{v} / \mathrm{v})$ ethanol to limit bacteria growth. Usually this stock dispersion of fd had a concentration of $20 \mathrm{mg} \mathrm{mL} \mathrm{m}^{-1}$ and was kept refrigerated at $+5{ }^{\circ} \mathrm{C}$. The concentration was determined photometrically using an extinction coefficient of $3.84 \mathrm{mg}^{-1} \mathrm{~cm}^{2}$ at $269 \mathrm{~nm}^{[16]}$. The absorption ratio at $269 \mathrm{~nm}$ and $244 \mathrm{~nm}\left(\mathrm{~A}_{269} / \mathrm{A}_{244}\right)$ was used to assess the purity of $\mathrm{fd}$, where a ratio of 1.41 indicates impurities below $1 \%$.

The isoelectric point (IEP) of $\mathrm{fd}$ is $4.2^{[30]}$, which means that fd is highly negatively charged at neutral or higher $\mathrm{pH}$, while it is positively charged if the $\mathrm{pH}$ is less than 4.2 . The coating procedures were carried out at low $\mathrm{pH}$ where $\mathrm{fd}$ is positively charged. The $\mathrm{pH}$ of the dispersions just before adding the silica precursor was measured and defined as the value for the coating, but it is noted that the $\mathrm{pH}$ after coating was about 0.2 units 
higher. For all tests the concentration of fd was kept in the range of the isotropic phase. Flow birefringence of dispersions was checked between crossed polarizers. TEOS (or THEOS) was added quickly under fast stirring, after which the dispersion was left standing for 12 hours under slow stirring. The amount of precursor was estimated assuming that a homogeneous silica layer with $1 \mathrm{~nm}$ thickness covering the whole fd surface will form after coating. For example, a stock dispersion of pure fd $(0.30 \mathrm{~mL}, \sim 20$ $\mathrm{mg} \mathrm{mL}^{-1}$ in $10 \mathrm{mM} \mathrm{NaHCO} 3$ buffer with $15 \% \mathrm{v} / \mathrm{v}$ ethanol) was diluted with the prediluted $\mathrm{HCl}(0.70 \mathrm{~mL}, \mathrm{pH} \sim 1.64)$. The resulting dispersion: $\sim 6 \mathrm{mg} \mathrm{mL}^{-1}, \mathrm{pH} \sim 2.32$, flow birefringent. To this dispersion, TEOS $(75 \mu \mathrm{L})$ was added under strong stirring. This dispersion was left standing for 12 hours before TEM analysis or any other processing of the sample.

Further treatment of some of the final dispersions after silica coating was done as follows: $0.5 \mathrm{~mL}$ of the dispersion after silica coating was mixed with $0.5 \mathrm{~mL}$ of a mixture of ammonia $(2.5 \mathrm{~mL})$, methanol $(18 \mathrm{~mL})$ and $\mathrm{H}_{2} \mathrm{O}(4.5 \mathrm{~mL})$ under stirring. TEM analysis of the resulting mixture was performed after 12 hours or later.

TEM was performed with a Philips EM200 transmission electron microscope operating at an accelerating voltage of $200 \mathrm{kV}$. Depending on the final status of the dispersion after silica coating, dilution with pure $\mathrm{H}_{2} \mathrm{O}$ by a factor $1 / 5 \sim 1 / 20$ was done and samples for TEM were prepared by dropping and evaporating the (diluted) dispersion onto a carboncoated copper grid. Purification of the dispersion was not carried out systematically because some samples washed with water by centrifugation showed no distinguishable difference under TEM compared to the samples without purification. 


\section{References}

[1] Y. N. Xia, P. D. Yang, Y. G. Sun, Y. Y. Wu, B. Mayers, B. Gates, Y. D. Yin, F. Kim, Y. Q. Yan, in Advanced Materials, Vol. 15, 2003, 353.

[2] G. R. Patzke, F. Krumeich, R. Nesper, Angew. Chem.-Int. Edit. 2002, 41, 2446.

[3] K. J. C. van Bommel, A. Friggeri, S. Shinkai, Angew. Chem.-Int. Edit. 2003, 42, 980.

[4] M. Sumper, E. Brunner, Advanced Functional Materials 2006, 16, 17.

[5] W. Shenton, T. Douglas, M. Young, G. Stubbs, S. Mann, Advanced Materials 1999, 11, 253.

[6] S. W. Lee, S. K. Lee, A. M. Belcher, Advanced Materials 2003, 15, 689.

[7] C. E. Flynn, S. W. Lee, B. R. Peelle, A. M. Belcher, Acta Mater. 2003, 51, 5867.

[8] E. Dujardin, C. Peet, G. Stubbs, J. N. Culver, S. Mann, Nano Letters 2003, 3, 413.

[9] N. Fujita, M. Asai, T. Yamashita, S. Shinkai, Journal of Materials Chemistry 2004, 14, 2106.

[10] M. J. Pender, L. A. Sowards, J. D. Hartgerink, M. O. Stone, R. R. Naik, Nano Letters 2006, 6, 40.

[11] Q. M. Ji, R. Iwaura, T. Shimizu, Chem. Lett. 2004, 33, 504.

[12] Q. Ji, R. Iwaura, M. Kogiso, J. H. Jung, K. Yoshida, T. Shimizu, Chem. Mat. 2004, 16, 250.

[13] S. S. Jong Hwa Jung, Toshimi Shimizu,, The Chemical Record 2003, 3, 212.

[14] J. H. Jung, S. Shinkai, Gels as Templates for Nanotubes, 2004.

[15] M. Numata, K. Sugiyasu, T. Hasegawa, S. Shinkai, Angew. Chem.-Int. Edit. 2004, 43, 3279.

[16] S. A. Berkowitz, L. A. Day, J. Mol. Biol. 1976, 102, 531.

[17] D. A. Marvin, Curr Opin Struc Biol 1998, 8, 150.

[18] J. Sambrook, D. W. Russell, Molecular Cloning: A Laboratory Manual, Cold Spring Harbor Laboratory Press, New York 2001.

[19] Z. Dogic, S. Fraden, in Soft Matter, Volume 2: Complex Colloidal Suspensions., (Eds: G. Gompper, M. Schick), WILEY-VCH Verlag GmbH \& Co. KGaA, Weinheim, Germany 2006, 1.

[20] P. J. Yoo, K. T. Nam, J. F. Qi, S. K. Lee, J. Park, A. M. Belcher, P. T. Hammond, Nat Mater 2006, 5, 234.

[21] C. B. Mao, D. J. Solis, B. D. Reiss, S. T. Kottmann, R. Y. Sweeney, A. Hayhurst, G. Georgiou, B. Iverson, A. M. Belcher, Science 2004, 303, 213.

[22] K. T. Nam, D. W. Kim, P. J. Yoo, C. Y. Chiang, N. Meethong, P. T. Hammond, Y. M. Chiang, A. M. Belcher, Science 2006, 312, 885.

[23] Y. Huang, C. Y. Chiang, S. K. Lee, Y. Gao, E. L. Hu, J. De Yoreo, A. M. Belcher, Nano Letters 2005, 5, 1429.

[24] M. Knez, M. Sumser, A. M. Bittner, C. Wege, H. Jeske, T. P. Martin, K. Kern, Advanced Functional Materials 2004, 14, 116.

[25] W. L. Liu, K. Alim, A. A. Balandin, D. M. Mathews, J. A. Dodds, Applied Physics Letters 2005, 86.

[26] S. Y. Lee, J. W. Choi, E. Royston, D. B. Janes, J. N. Culver, M. T. Harris, J. Nanosci. Nanotechnol. 2006, 6, 974. 
[27] Y. A. Shchipunov, T. Y. Karpenko, Langmuir 2004, 20, 3882.

[28] R. A. Caruso, M. Antonietti, Chem. Mat. 2001, 13, 3272.

[29] K. J. C. van Bommel, S. Shinkai, Langmuir 2002, 18, 4544.

[30] K. Zimmermann, H. Hagedorn, C. C. Heuck, M. Hinrichsen, H. Ludwig, J Biol Chem 1986, 261, 1653. 


\section{Figures}
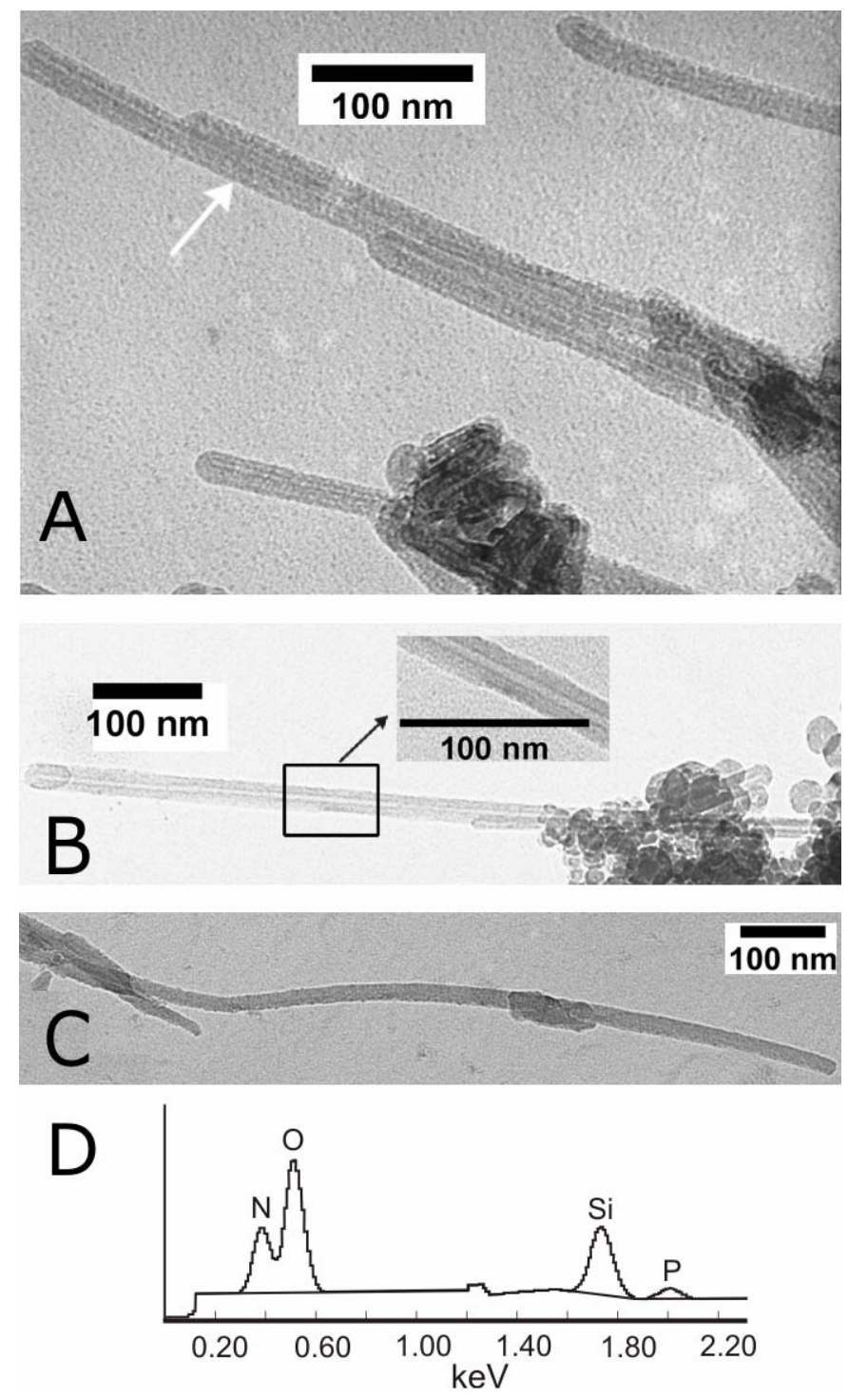

Figure 1 TEM images of typical rods. (A) rods with a uniform silica layer and semi-spherical ends, an assembly of three rods is indicated by the arrow; (B) a rod with a clear core-shell structure; (C) a slightly curved rod where no core-shell structure is visible; (D) EDAX analysis of the rod shown in $\mathrm{C}$. 


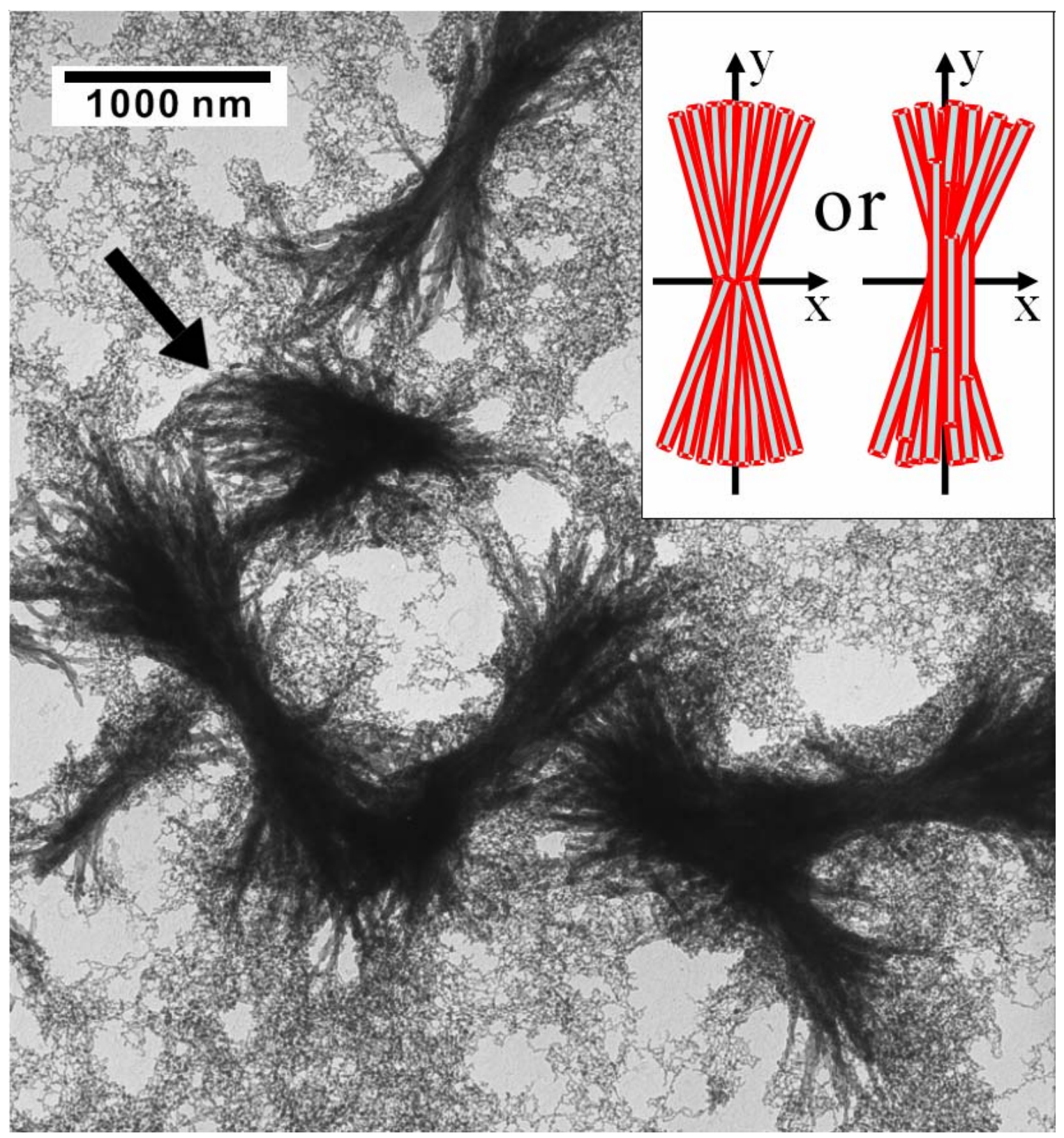

Figure 2 TEM images of bow-tie-shaped bundles dispersed in the background of granular silica.

A possible subunit of the bundles, a silica fan is indicated by the arrow. Inset: schematic drawings of possible structures of the bundles. 

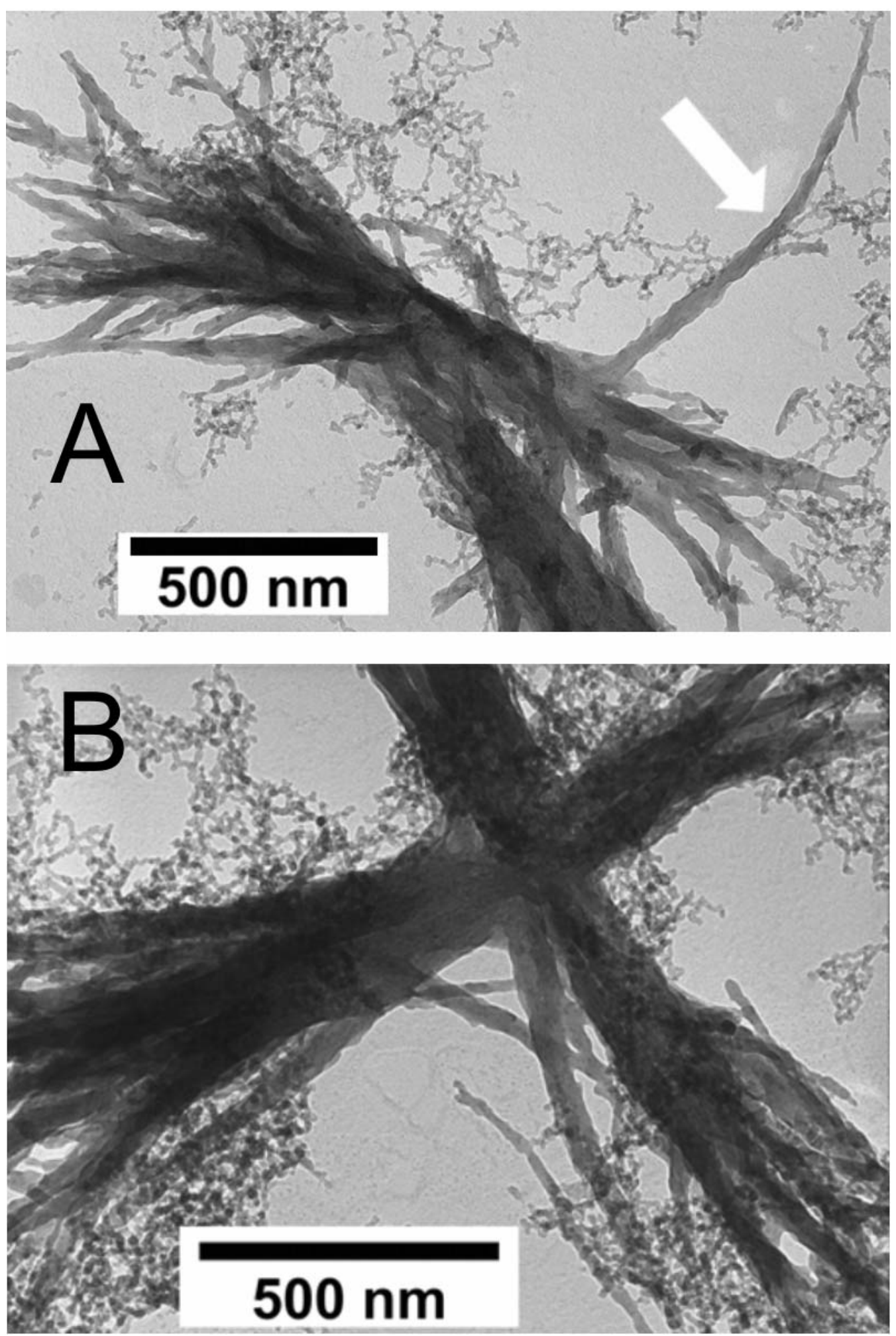

Figure 3 TEM images of bow-tie-shaped bundles with specific morphologies. (A) A defect bundle with a single rod protruding as indicated by the arrow; (B) a cross-like bundle. 

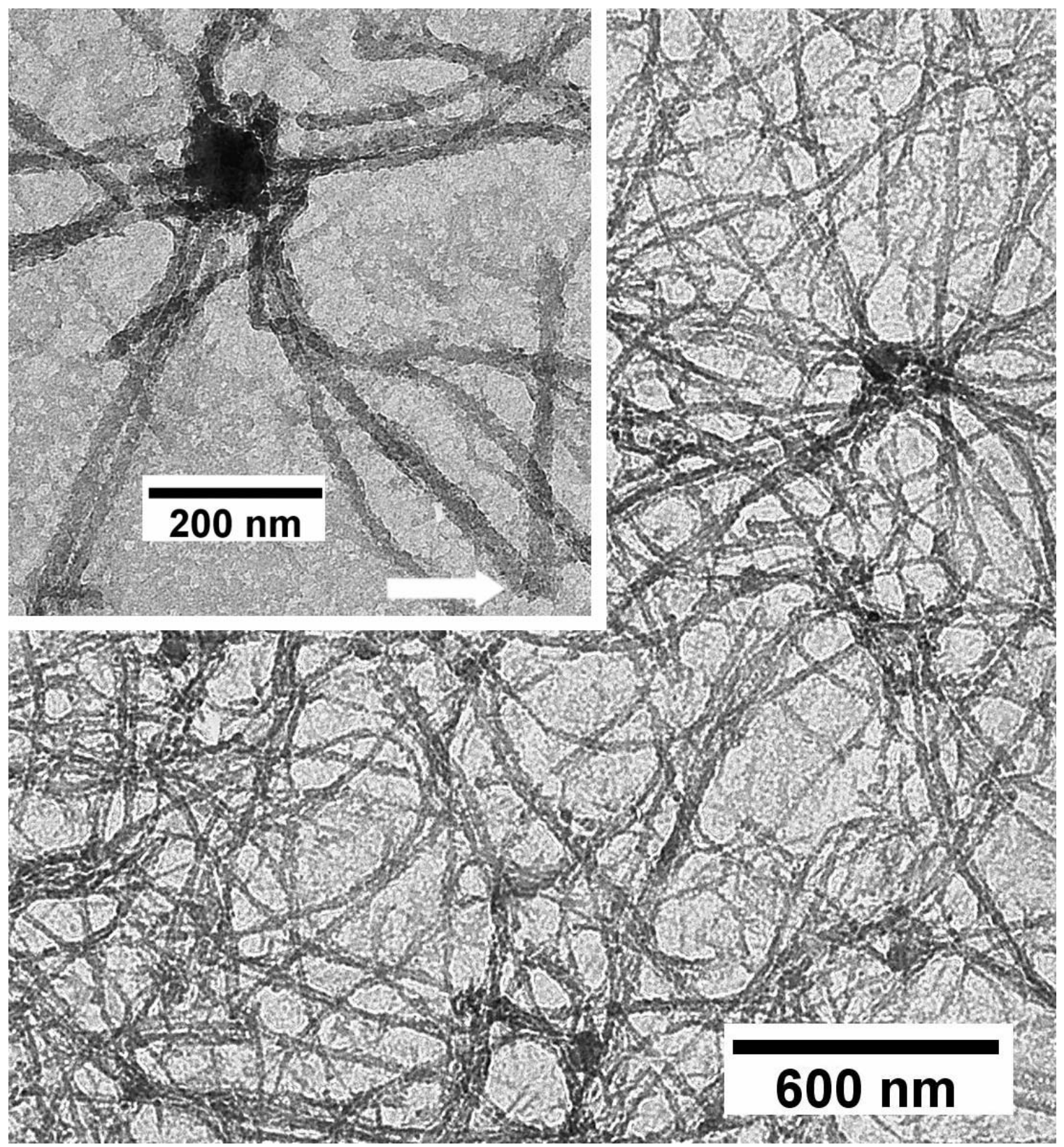

Figure 4 TEM of nanowires. A broken wire can be seen in the inset as indicated by the arrow. 

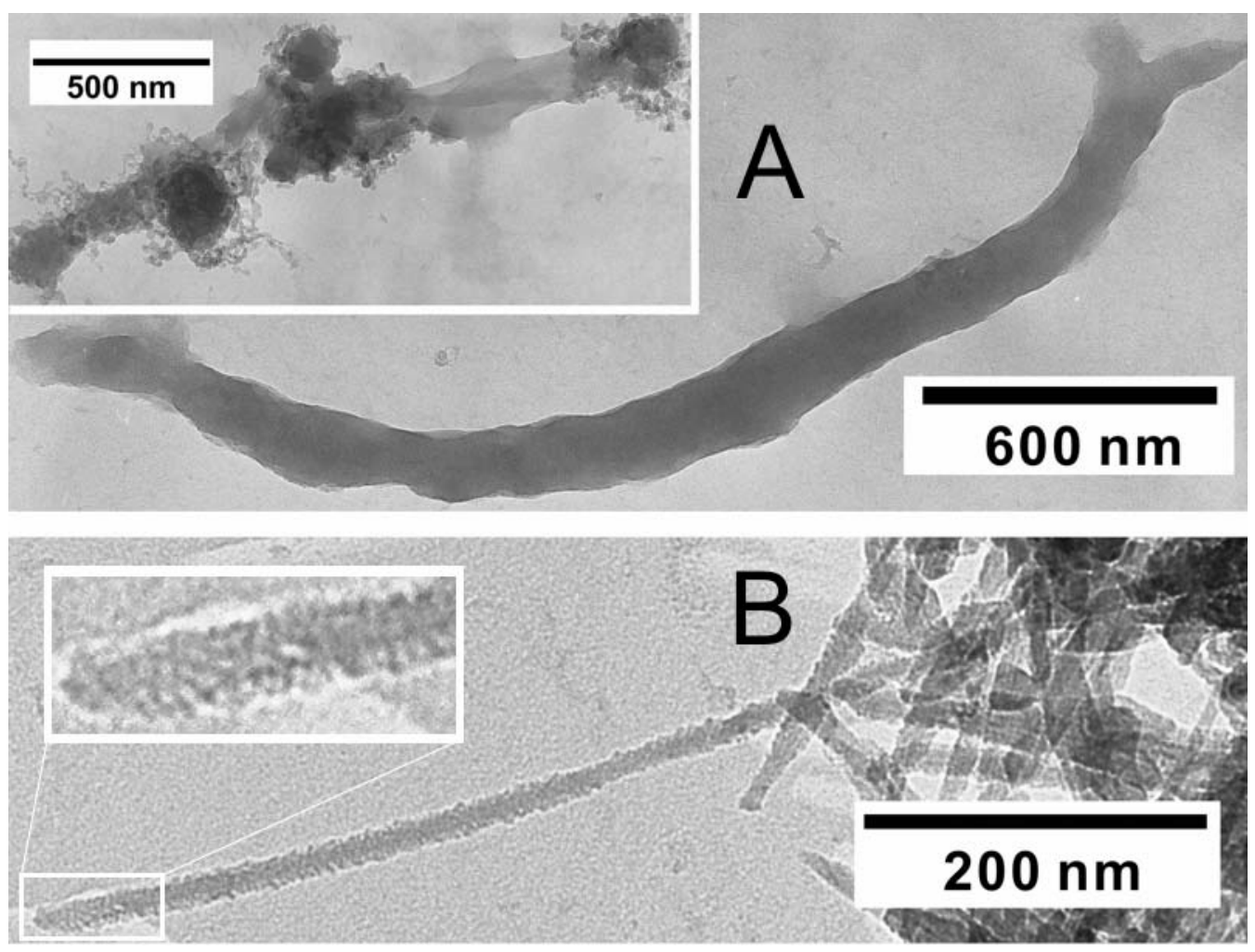

Figure 5 TEM of typical morphologies of less well-defined silica rods. (A) Big rods when

THEOS was used as silica precursor. Inset: two big rods with a length comparable to that of fd; (B) Small rods with rough surface when some ethanol was added in combination with TEOS as precursor. Inset: the magnified version of the end part of the rod. 


\section{Keywords}

Template, fd virus, silica, rods, nanostructures

\section{Table of content abstract}

We explored fd as a template to direct the formation of silica nanomaterials with different morphologies through simple sol-gel chemistry. Depending on the conditions silica nanowires can be formed, which seem to accurately transcript the bending conformation and the length of the fd viruses in solution. But also surprisingly straight silica rods may be formed, and under other conditions bow-tie-shaped bundles of rods are formed, which have a remarkably well defined shape and dimension. 